\title{
Blood Lactate Level as Predictor of Early Outcome after Off-Pump Coronary Artery Bypass Grafting
}

\author{
Nasrin Aktar ${ }^{3}$, AM Asif Rahim², AYM Shahidullah ${ }^{3}$, AHM Iftekher Hossain², Farooque Ahmed ${ }^{1}$ \\ ${ }^{1}$ Department of Cardiac Surgery, National Heart Foundation Hospital and Research Institute, \\ Dhaka, ${ }^{2}$ Department of Cardiac Surgery, Chattogram Medical College Hospital, \\ Chattogram, ${ }^{3}$ Department of Cardiac Surgery, Khulna City Medical college, Khulna
}

\begin{abstract}
Key Words : Blood Lactate Level, Coronary Artery Bypass grafting, Ischaemic heart disease.
\end{abstract}

\begin{abstract}
:
Background: Identification of predictors of morbidity and mortality is an important issue for the management of cardiac surgical patients. Elevated lactate concentrations in the immediate postoperative period reflect unmet metabolic demand and may be associated with outcome.

Methods: A prospective observational study was carried out in the department of cardiac surgery, at National Heart Foundation Hospital\& Research Institute(NHFH\&RI), Dhaka. commencing from July 2016 to March 2018. In all Adult patients irrespective of sex undergoing elective off-pump coronary artery bypass grafting $(O P C A B G)$. Purposive sampling was applied with patient with blood lactate level $<3 \mathrm{mmol} / \mathrm{L}$ 6hours after shifting from OT to ICU in Group-A and blood lactate level $\geq 3 \mathrm{mmol} / \mathrm{l}$, 6hours after shifting to the ICU in Group-B.Postoperative early adverse outcomes were analyzed in both the patient groups.

Results: Blood lactate level 6 hours after ICU transfer is an independent predictor for prolonged mechanical ventilation time, (OR 2.540,95\% CI 1.781-3.621, $p=<0.001)$.prolonged ICU stay, .(OR 1.767, 95\% CI 1.348-2.315, $p=<0.001$ ) pulmonary complication (OR 1.503, 95\% CI 1.104-2.046, $p=$ 0.010). and renal dysfunction(OR 1.460, 95\% CI 1.156-1.843, $p=0.001)$. following off-pump coronary artery bypass grafting. Other risk factors for adverse outcome including, low LVEF, diabetes mellitus, blood transfusion and inotropic support were also found to be predictors of major complications in our study.

Conclusion: The findings of this study permits to conclude that blood lactate level 6hours after ICU transfer is associated with an increased risk of postoperative early adverse outcome after off-pump coronary artery bypass grafting. It is found that lactate is an independent predictor of major postoperative complications. So, achieving normal blood lactate level following OPCABG is essential for minimizing postoperative adverse outcome.
\end{abstract}

(Cardiovasc. j. 2020; 12(2): 120-125)

\section{Introduction:}

Identification of predictors of morbidity and mortality is an important issue for the management of cardiac surgical patients. Morbidity and mortality are directly related to the preoperative status of the patient, as well as to specific anesthetic, surgical and postoperative factors. After coronary artery bypass grafting
(CABG) surgery, hyperlactatemia (HL) is frequently seen in $10-20 \%$ patients. Elevated lactate concentrations in the immediate postoperative period reflect unmet metabolic demand and may be associated with outcome. ${ }^{1}$

There is overwhelming evidence that sepsis and septic shock are associated with hyperlactatemia (sepsis-associated hyperlactatemia). Sepsis

Address of Correspondence: Dr. Nasrin Aktar, Dept. of Cardiac Surgery, Khulna City Medical college, Khulna Bangladesh. Email-nasrinsomc42@gmail.com

- 2020 authors; licensed and published by International Society of Cardiovascular Ultrasound, Bangladesh Chapter and Bangladesh Society of Geriatric Cardiology. This is an Open Access article distributed under the terms of the CC BY NC 4.0 (https://creativecommons.org/licenses/by-nc/4.0). 
associated hyperlactatemia (SAHL) has been recently promoted as a way of identifying patients with 'cryptic'shock who require focused, early goaldirected therapy. ${ }^{2}$

Pyruvate is the intermediate metabolite of glycolysis. Under aerobic condition, pyruvate is converted to acetyl co-A to enter the Kreb's cycle. In anaerobic conditions, pyruvate is converted by lactate dehydrogenase (LDH) to lactic acid. That's how; lactate represents a useful and clinically obtainable marker of tissue hypoxia also. ${ }^{3}$

Lactate can be metabolized by the liver and the kidney either by direct oxidation or as a source of gluconeogenesis (Cori cycle). Hepatocytes are the major site of oxidative lactate uptake but the kidneys account for approximately $30 \%$ of lactate metabolism. ${ }^{13}$ Approximately half of available lactate is disposed of via oxidation at rest and maintains a constant lactate to pyruvate ratio of approximately 10:1. Lactate clearance has been estimated at an extraordinary value of 800-1800 $\mathrm{ml} / \mathrm{min}$ by studying the disposal of infused sodium L-lactate. ${ }^{2,3}$

Hajjar et al. shows that, blood lactate level $>3$ $\mathrm{mmol} / \mathrm{l}$ during cardio-pulmonary bypass (CPB) and 6 hours after transfer in ICU is associated with an increased risk of post-operative morbidity and mortality. It was found to be an independent predictor of major post-operative complications including prolong mechanical ventilation time, prolong ICU stay, pulmonary complication, renal complication and mortality. ${ }^{4}$

In both surgical and nonsurgical settings like infection, burn, trauma, sepsis and following cardiopulmonary resuscitation elevated lactate concentration has been shown to correlate with mortality. Recently magnesium and thiamine are being used for lowering the blood lactate level as magnesium plays an important role in oxidative metabolism. In the absence of magnesium, disrupted oxidative phosphorylation shifts toward anaerobic metabolism. ${ }^{5}$

Coronary revascularization using cardiopulmonary bypass remains the gold standard treatment for coronary artery disease. Over the past decade, techniques of revascularization on the beating heart without the use of cardiopulmonary bypass (off-pump surgery) have evolved with an attempt to reduce the potential deleterious effects of extracorporeal circulation. Several trials have reported a significant reduction in morbidity with avoidance of cardiopulmonary bypass, while large observational studies have also reported a reduction in mortality. ${ }^{6}$

Off-pump surgery requires stabilization of the heart and good exposure of the target place of the anastomosis. In the period of performing distal anastomosis, reduction of the blood pressure caused by handling of the heart usually occurs. As a consequence, there could be reduction of the blood supply in the peripheral tissue. Changes in tissue metabolic activity could influence the postoperative clinicaloutcomes. ${ }^{7}$

Hemodynamic derangement during displacement of beating heart in off-pump coronary artery bypass graft (OPCAB) surgery might be related with right ventricular (RV) dysfunction. A few studies on hemodynamic alternations associated with $\mathrm{OPCAB}$ reported that reduced functions of both ventricles during coronary artery anastomosis are the main mechanism of hemodynamic derangements and especially, impaired diastolic function of the right ventricle $(\mathrm{RV})$ plays an important role. ${ }^{8}$

Postoperative hyperlactatemia has been shown to correlate with increased postoperative morbidity. Persistent lactate production after reperfusion reflects delayed recovery of aerobic metabolism. Hence monitoring of postoperative lactate levels may be useful for assessing the status of aerobic metabolism. ${ }^{9}$ Mean lactate concentrations during the first 12 hours of CVICU admission had the next best predictive ability. 1,9

Recent literatures demonstrate that elevated lactate concentrations and prolong lactate clearance time are associated with increased morbidity and death in patients undergoing CABG or valve operations or both. Timely monitoring of postoperative lactate levels and correction of hyperlactatemia may facilitate rapid recovery of aerobic metabolism and reduce postoperative complications. Thus, this biochemical marker may provide clinicians with an early indication of a patient's likelihood of experiencing major complications even after off-pump coronary artery revascularization. ${ }^{1,9}$ 


\section{Methods:}

This Prospective observational study was carried out in the Department of Cardiac Surgery, at National Heart Foundation Hospital\& Research Institute (NHFH\&RI), Dhaka from July 2016 to March 2018. The study was carried out in all adult patients undergoing elective OPCAB surgery and fulfill the inclusion and exclusion criteria with. Inclusion criteria - adult patients irrespective of sex undergoing elective off-pump coronary artery bypass grafting. Exclusion criteria were high lactate before induction $(>2 \mathrm{mmol} / \mathrm{L})$, emergency surgery-do surgery-exploration for bleeding, hepatic and renal dysfunction, conversion from OPCAB to on-pump bypass grafting, intraoperative mortality or mortality less than 6 hours after ICU transfer. Consecutive type was followed for sampling technique. Patients were divided into two groups according to the blood lactate level 6 hours after transfer in ICU following OPCAB. Group - A - patients who have blood lactate $<3 \mathrm{mmol} / \mathrm{L}$, 6 hours after shifting to the ICU; Group-B - patients who have blood lactate $\geq 3 \mathrm{mmol} / \mathrm{l}, 6$ hours after shifting to the ICU. Post-operative variables includes Lactate ( $\mathrm{mmol} / \mathrm{L}$ ) after transfer in ICU (0 hours after transfer in ICU, 6 hours after transfer in ICU, 12 hours after transfer in ICU), blood transfusion units, postoperative blood loss, mechanical ventilation time, duration of ICU stay, low output syndrome(requirement of inotropes $>24$ hours), post-operative IABP requirement, postoperative complications- (Neurological deficit, pulmonary complication, arrhythmia, renal dysfunction, mortality).

Five arterial blood samples had been drawn from each patient: just before the anesthetic induction, at the end of coronary revascularization, immediately after transfer in ICU (0 hour), 6 hours and 12 hours after transfer in ICU. Arterial blood samples were analyzed in the ICU by arterial blood gas analyzer, model_TechnoMedica, gastat-603 i.e. blood gas system. Data were collected using the predesigned case record form (CRF). Both pre and post-operative variables were collected before discharge and post-operative complications after discharge were recorded during follow up.

Data were analyzed by software statistical program for social science (SPSS-20). Statistical analysis was done by using chi-square test and /or fisher's exact test for qualitative data; t-test and Mann-Whitney $\mathrm{U}$ test were done for quantitative data. Logistic regression analysis was performed to identify significant predictors of outcome using variables. Continuous variables were shown as mean \pm standard deviation (SD) and categorical variables were given as number. A p-value of $<0.05$ were considered as significant.

\section{Results:}

A total number of 150 patients undergoing $\mathrm{OPCAB}$ were recruited for this study and divided into two groups according to the level of lactate 6 hours after transfer in the ICU. Regarding demographic variables majority of patients were in $55-65$ years age group which is $21(45.7 \%)$ patients and $44(42.3 \%)$ patients in group A and group B respectively. Only $4(8.7 \%)$ in group $\mathrm{A}$ and $9(8.7 \%)$ patients in group B were more than 65 years aged $(\mathrm{p}=0.338)$. Males are more in both groups, which are $43(93.5 \%)$ patients and 93(89.4\%) patients in group A and group B respectively ( $\mathrm{p}=0.552) .20(43.5 \%)$ over weight patients in group A and 55 (52.9\%) in group B. Obese patients were $4(8.7 \%)$ and $7(6.7 \%)$ in group $A$ and group $B(p=0.605)$.

Baseline clinical parameters distribution among the study population. Here, good left ventricular ejection fraction $(52.30 \pm 5.49 \%)$ found in patients of group A and moderate left ventricular ejection fraction $(49.12 \pm 8.40 \%)(p=0.007)$. Majority of the patients are having triple vessels disease. 37(80.4\%) patients in group A and 79(76.0\%) in group B have triple vessel disease $(p=0.804)$. History of myocardial infarction present in $30(65.2 \%)$ patients and $79(76.0 \%)$ patients in group A and in group B respectively $(p=0.173)$.

Regarding peroperative variables total operative time Table I reveals the mean operative time in

Table-I

Comparison oftotal operative timeof the study population( $n=150)$.

\begin{tabular}{lccc}
\hline Variables & \multicolumn{2}{c}{ Lactate Group } & p value $^{\text {c }}$ \\
\cline { 2 - 3 } & Group A $(<3)(\mathrm{n}=46)$ & Group B $(\mathrm{e}$ "3)(n=104) & \\
\hline Total operative time & $312.83 \pm 51.84$ & $351.15 \pm 49.27$ & $<0.001$ \\
\hline
\end{tabular}

$c_{t}$ test was done to measure the level of significance $(p=0.05)$. 
group A and group B is $312.83 \pm 51.84 \mathrm{~min}$ and $351.15 \pm 49.27 \mathrm{~min}$ respectively $(\mathrm{p}=<0.001)$.

Majority of patients received 1 unit blood. 41(89.1\%) patients and $85(81.7 \%)$ in group A and in group B respectively received 1 unit blood $(\mathrm{p}=0.024) .3$ grafts were done in $28(60.9 \%)$ patients and $33(31.7 \%)$ in group A and group B respectively. 4 grafts were received by $13(28.3 \%)$ patients in group A and 48 (46.2\%) in group B. Mean \pm SD of graft done in two groups are $3.43 \pm 0.69$ and $3.63 \pm 0.87$ $(\mathrm{p}=0.171) .10(21.7 \%)$ patients in group A required inotropes while $41(39.4 \%)$ patients needed inotropes peroperatively $(\mathrm{p}=0.035)$.

Table II shows serial blood lactate levels per and postoperatively in both groups. At $6^{\text {th }}$ hours patients of group A have mean blood lactate $2.05 \pm 0.37 \mathrm{mmol} /$ $\mathrm{L}$ and $5.02 \pm 1.62 \mathrm{mmol} / \mathrm{L}$ in group $\mathrm{B}(\mathrm{p}<0.001)$.

Table III shows postoperative variables in terms of mechanical ventilation time (min), duration of ICU stay (hours), blood loss (ml), mean mechanical ventilation time between two groups is $402.17 \pm 92.88 \mathrm{~min}$ and $533.08 \pm 181.27 \mathrm{~min}$ respectively $(p<0.001)$. Mean duration of ICU stay in group $\mathrm{A}$ is $38.98 \pm 12.91$ hours and $66.11 \pm 43.51$ hours in group B $(\mathrm{p}<0.001)$. Mean blood loss between patients in group A is $85.43 \pm 81.12 \mathrm{ml}$ and in group $B$ is $107.31 \pm 63.89(p=0.001)$.

Low output syndrome was found in $65(53.8 \%)$ patients in group B. None of the patient in group A needed inotropes for $>24$ hours $(p<0.001)$. Units of blood transfused postoperatively among the study populations. $26(56.5 \%)$ patients in group A and 37 (35.6\%) in group B did not received any blood transfusion postoperatively. 1 unit blood was transfused to the $19(41.3 \%)$ patients in group A and 61(58.7\%) patients in group B ( $p=0.099)$.

Neurological deficit was found in $0(0 \%)$ patient in group A and in 7 (6.7\%) in group B ( $p=0.101) .10$ $(9.6 \%)$ patients in group B developed arrhythmia postoperatively $(p=0.032)$. There was no mortality in group $\mathrm{A}$ and $2(1.9 \%)$ in group $\mathrm{B}(\mathrm{p}=0.999)$.

Pulmonary complications found in $1(2.2 \%)$ patient and in 20 (19.2\%) patients in group A and group B respectively $(\mathrm{p}=0.006)$. Renal dysfunction found in $4(8.7 \%)$ patient in group $\mathrm{A}$ and $43(41.3 \%)$ in group B $(\mathrm{p}=<0.001)$.

Table-II

Comparison of blood lactate levels in two groups at different timing $(n=150)$.

\begin{tabular}{lccc}
\hline Blood lactate & \multicolumn{2}{c}{ Lactate Group } & p value $^{\mathrm{c}}$ \\
\cline { 2 - 3 } & Group A $(<3)(\mathrm{n}=46)$ & Group B $(\geq 3)(\mathrm{n}=104)$ & \\
\hline Before the anesthetic induction & $0.91 \pm 0.22$ & $1.18 \pm 0.82$ & 0.031 \\
At the end of revascularization & $2.30 \pm 0.59$ & $3.53 \pm 1.06$ & $<0.001$ \\
Immediately after transfer in ICU (0 hour) & $2.47 \pm 0.93$ & $3.95 \pm 1.30$ & $<0.001$ \\
6 hours after transfer in ICU & $2.05 \pm 0.37$ & $5.02 \pm 1.62$ & $<0.001$ \\
12 hours after transfer in ICU & $1.65 \pm 0.51$ & $4.48 \pm 1.97$ & $<0.001$ \\
\hline
\end{tabular}

$c_{t}$ test was done to measure the level of significance $(p=0.05)$.

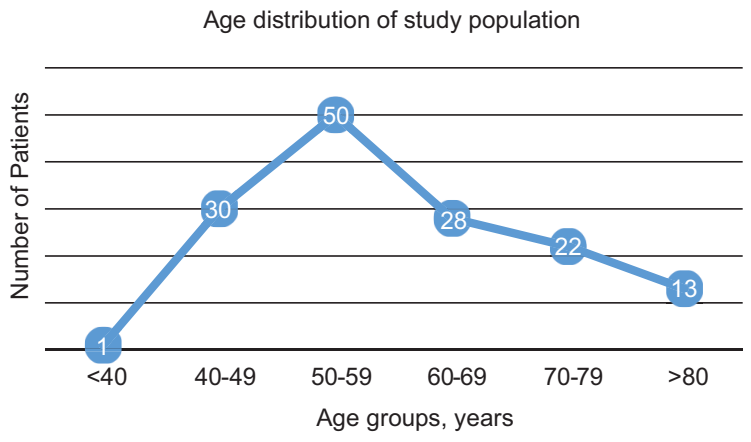

Fig.1: Line diagram of blood lactate levels in different time shows persistent high lactate level in group $B$ population $(n=150)$. 
Table-III

Comparison of postoperative variables $(n=150)$.

\begin{tabular}{lccc}
\hline Postoperative variables & \multicolumn{2}{c}{ Lactate Group } & \multirow{2}{*}{ p value $^{\mathrm{d}}$} \\
\cline { 2 - 3 } & Group A $(<3)$ & Group B $(\geq 3)$ & \\
\hline Mechanical ventilation time (min) & $402.17 \pm 92.88(46.74)$ & $533.08 \pm 181.27(88.22)$ & $<0.001$ \\
Duration of ICU stay (hours) & $38.98 \pm 12.91(46.20)$ & $66.11 \pm 43.51(88.46)$ & $<0.001$ \\
Blood loss (ml) & $85.43 \pm 81.12(58.37)$ & $107.31 \pm 63.89(83.08)$ & 0.001 \\
\hline
\end{tabular}

${ }^{d}$ Mann-Whitney $U$ test was done to measure the level of significance $(p=0.05)$.

Figure within parentheses indicates in Mean Rank.

In the logistic regression analysis, blood lactate 6 hours after transfer in ICU was found to be an independent predictor of prolonged VT. (OR 2.540, $95 \%$ CI 1.781-3.621, $\mathrm{p}=<0.001$ ).

Blood lactate 6 hours after transfer in ICU was found to be an independent predictor of prolong ICU stay, pulmonary complication \& renal dysfunction. Blood lactate 6hours after transfer in ICU was not found to be an independent predictor of postoperative inotropic support, postoperative neurological deficit, arrhythmia and mortality.

\section{Discussion :}

This study has demonstrated that a lactate level $\geq 3 \mathrm{mmol} / \mathrm{L}$ at $6^{\text {th }}$ hour after ICU transfer is related to postoperative adverse events and is independently associated with risk of major complications after off-pump coronary bypass grafting.

Majority of patients were in 55-65 years age group. Males are more in both groups. The differences between the two groups (age, sex, BMI) is statistically not significant.The co-morbidities were recorded among the study population. Differences between the two groups for hypertension, dyslipidemia, cerebrovascular disease were statistically not significant. Prevalence of DM was more in Group B. Hyperlactemia is associated with hyperglycaemia. ${ }^{3,10,11}$ Good left ventricular ejection fraction $(52.30 \pm 5.49 \%)$ found in patients of group A and moderate left ventricular ejection fraction (49.12 $\pm 8.40 \%$ ) in Group B. Similar result was seen in the study conducted by Hajjar, et al. and Maillet, et al. ${ }^{4,10}$

Outcome after off-pump coronary artery bypass grafting is determined by preoperative characteristics (diabetes, low LVEF) as stated above in addition to preoperative factors such as total operative time, units of blood transfusion and peroperative inotropes requirements. Increased number of blood transfusion is found to be a risk factor for high lactate. ${ }^{4}$ In this study peroperative requirements of inotropic support and prolong operative time were found associated with high blood lactate level. Similar result was found in other studies. ${ }^{3,10,11}$ Comparison of mean blood lactate level at different point of time between the two groups shows significant differences at before induction, at the end of revascularization, immediately after transfer in ICU, at $6^{\text {th }}$ hours after transfer in ICU, at $12^{\text {th }}$ hours after transfer in ICU. Similarly, Lindsey et al., stated that persistent high lactate level is a risk factor of postoperative complications. Hajjar, et al., concluded that a lactate level exceeding $3 \mathrm{mmol} / \mathrm{L}$ at 6 hours after surgery is independentlyassociated with a 3.3 times risk of a major complication after cardiac surgery, including death. ${ }^{4}$ The present study showed, mean mechanical ventilation time between two groups is $402.17 \pm 92.88 \mathrm{~min}$ and 533.08 \pm 181.27 min respectively with significance $(\mathrm{p}<$ 0.001). Patients with high blood lactate need prolong mechanical ventilation time. ICU care was needed in group A for $38.98 \pm 12.91$ hours and 66.11 \pm 43.51 hours in group B. Maillet, et al., Lindsey, et al., and Hajjer, et al., showed in different studies that prolong mechanical ventilation and ICU stay, found in patients with hyperlactatemia and was statistically significant. ${ }^{1,4,10}$ Postoperative blood loss is associated with hyperlactatemia and early adverse outcome.${ }^{1}$ In this study low cardiac output syndrome was clarified as the requirement of inotropes for $>24$ hours. Here, in group A no patient required inotropes postoperatively for $>24$ hours, but 56 (53.8\%) patients in group B needed inotropes for $>24$ hours. Similar results were seen in the other studies. ${ }^{7,10,13}$ Outcome variables in 
terms of neurological deficit, pulmonary complication, arrhythmia, renal dysfunction and mortality were recorded. There was no difference in neurological deficit and mortality. Pulmonary complications, renal dysfunction and postoperative arrhythmia were more in Group B.

The study done by Hajjer, et al., showed that hyperlactatemia 6 hours after ICU admission is an independent risk factor for worse outcome after cardiac surgery. ${ }^{16}$ In our study, binary logistic regression analysis shows that blood lactate level 6 hours after ICU transfer is an independent predictor for prolonged mechanical ventilation time,pulmonary complication and renal dysfunction. Other risk factors for adverse outcome including, low LVEF, diabetes mellitus, blood transfusion and inotropic support were also predictors of major complications in our patients, similar to previous studies. ${ }^{1,4,9}$ It is realized that hyperlactatemia is common both intraoperatively and postoperatively. Dynamic changes in lactate, accounting not only for the magnitude but also for the duration of hyperlactatemia is an independent predictor of early adverse outcome following OPCAB and normalization of lactate within 6 hours is associated with lower rate of complications. ${ }^{12}$

\section{Conclusion:}

The findings of this study permit to conclude that blood lactate level 6 hours after ICU transfer is associated with an increased risk of postoperative early adverse outcome after off-pump coronary artery bypass grafting. It is found that lactate is an independent predictor of major postoperative complications including prolong mechanical ventilation time, prolong ICU stay, pulmonary complication and renal dysfunction following offpump coronary artery bypass grafting. Other risk factors for adverse outcome including, low LVEF, diabetes mellitus, blood transfusion and inotropic support were also found to be predictors of major complications. So, achieving normal blood lactate level following OPCABG is essential for minimizing postoperative adverse outcome.

\section{Conflict of Interest - None.}

\section{References:}

1. Lindsay AJ, Xu M, Sessler DI, Blackstone EH, Bashour CA. Lactate clearance time and concentration linked to morbidity and death in cardiac surgical patients. Ann Thorac Surg 2013; 95: 486-492.

2. Garcia-Alvarez M, Marik P, Bellomo R. Sepsis-associated hyperlactatemia. Critical Care 2014 ; 18 : 503.

3. Phypers B, Pierce JMT. Lactate physiology in health and disease. CEACCP 2006 ; 26(3) : 128-132.

4. Hajjar LA, Almeida JP, Fukushima JT, Rhodes A, Vincent JL, Osawa EA, Galas FRBG. High lactate levels are predictors of major complications after cardiac surgery. J Thorac Cardiovasc Surg 2013; 146: 455-460.

5. Moskowitz A, Lee J, Donnino MW, Mark R, Celi LA, Danziger J. The association between admission magnesium concentrations and lactic acidosis in critical illness. J Intensive Care Med 2016; 31(3): 187-192.

6. Abu-Omar Y, Taggart DP. The present status of offpump coro-nary artery bypass grafting. Eur $J$ Cardiothorac Surg 2009 ; 36 :312-321.

7. Pojar M, Mand'ák J, Cibíèek N, Lonský V, Dominik J, Palièka V, et al. Peripheral tissue metabolism during off-pump versus on-pump coronary artery bypass graft surgery: the microdialysis study. Eur $J$ Cardiothorac Surg 2008 ; 33(5) : 899-905.

8. Kwak Y, Oh Y, Jung S, Yoo K, Lee J, Hong Y. Change in right ventricular function during off-pump coronary artery bypass graft surgery. Eur J Cardiothorac Surg 2004 ; 25(4) : 572-577.

9. Parissis H, Mbarushimana S, Ramesh BC, Parissis M, Lampridis S, Mhandu P, Al-Alao B. The impact of offpump surgery in end-organ function: practical endpoints. J Cardiothorac Surg 2015; 10(1): 159.

10. Maillet J-M, Le Besnerais P, Cantoni M. Frequency, risk factors, and outcome of hyperlactatemia after cardiac surgery. Chest 2003; 123: 1361-1366.

11. Totaro RJ, Raper RF. Epinephrine-induced lactic acidosis following cardiopulmonary bypass. Crit Care Med 1997; 25(10) : 1693-1699.

12. Sanfilippo F, Chiarenza F, Cassisi C, Santonocito C, Tsoutsouras T, Trivella M, et al. The Effects of OnPump and Off-Pump Coronary Artery Bypass Surgery on Metabolic Profiles in the Early Postoperative Period. $J$ Cardiothorac Vasc Anesth 2016 ; 30(4) : 909-916. 